\title{
Chronic AMPK inactivation slows SHH medulloblastoma progression by inhibiting mTORC1 signaling and depleting tumor stem cell populations
}

Taylor Dismuke ${ }^{* * 1}$, Daniel Shiloh Malawsky ${ }^{* * 1}$, Hedi Liu' ${ }^{1}$, Jay Brenman ${ }^{2,3}$, Andrey Tikunov ${ }^{1}$, Timothy R. Gershon*1,3,4

${ }^{1}$ Department of Neurology, University of North Carolina School of Medicine, Chapel Hill, NC 27599, USA

${ }^{2}$ Department of Cell Biology and Physiology, University of North Carolina School of Medicine, Chapel Hill, NC 27599 USA

${ }^{3}$ UNC Neuroscience Center, University of North Carolina School of Medicine, Chapel Hill, NC 27599, USA

${ }^{4}$ Lineberger Comprehensive Cancer Center, University of North Carolina School of Medicine, Chapel Hill, NC 27599, USA email: gershont@neurology.unc.edu

* corresponding author

${ }^{* *}$ contributed equally to the work

Running title: AMPK in medulloblastoma

TD was supported by NINDS (F31 NS120459). T.R.G. was supported by NINDS (R01NS088219, R01NS102627, R01NS106227).

Corresponding author: Timothy Gershon, MD, PhD, CB7025 UNC School of Medicine, Chapel Hill, NC 27599. Phone: (919) 9662528 FAX: (919) 966-1922 email:

gershont@neurology.unc.edu

The authors declare no conflicts of interest.

Word count: 4687 (including Methods, excluding Abstract and Figure Legends) \# of Figures: 5 


\section{Abstract}

We show that inactivating AMPK in vivo in a genetic model of medulloblastoma depletes tumor stem cell populations and slows tumor progression. Medulloblastoma, the most common malignant pediatric brain tumor, grows as heterogenous communities comprising diverse types of tumor and stromal cells. We have previously shown that different types of cells in medulloblastomas show different sensitivities to specific targeted therapies. To determine if specific populations depend on AMPK, we analyzed mice with AMPK-inactivated medulloblastomas. We engineered mice with brain-wide, conditional deletion of the AMPK catalytic subunits Prkaa1 and Prkaa2 and conditional expression SmoM2, an oncogenic Smo allele that hyperactivates Sonic Hedgehog $(\mathrm{SHH})$ signaling. We compared the medulloblastomas that formed in these mice to tumors that form in AMPK-intact mice with conditional SmoM2 expression. AMPKinactivated tumors progressed more slowly, allowing longer event-free survival. AMPK inactivation altered the cellular heterogeneity, determined by scRNA-seq, increasing differentiation, decreasing tumor stem cell populations and reducing glio-neuronal multipotency. Mechanistically, AMPK inactivation altered glycolytic gene expression and decreased mTORC1 pathway activation. Hk2-deletion reproduced key aspects of the AMPK-inactivation phenotype, implicating altered glycolysis in the tumor suppressive effect of AMPK inactivation. Our results show that AMPK inactivation impairs tumor growth through mechanisms that disproportionately affect tumor stem cell populations. As stem cells are intrinsically resistant to current cytotoxic therapy that drives recurrence, finding ways to target these populations may prevent treatment failure. Our 
data suggest that targeted AMPK inactivation may produce therapeutic effects in tumor stem cell populations refractory to other therapeutic approaches.

\section{Introduction}

Medulloblastoma, the most common malignant pediatric brain tumor, is a heterogeneous group of cancers with 4 major subgroups [1-4], and individual tumors in each subgroup show cellular heterogeneity $[5,6]$. In each subgroup, recurrence after treatment is the major cause of morbidity. Cellular heterogeneity may contribute to recurrence by increasing overall robustness to overcome selective pressure of therapy, and by generating specific resistant populations that drive recurrence. Understanding the impact of new therapies on the diversity of cell types within medulloblastomas is critical to designing therapies that can reduce the chance of recurrent disease.

SHH (Sonic Hedgehog) subgroup tumors, the most frequent subgroup of medulloblastoma, can be recapitulated in mice genetically engineered to hyperactivate $\mathrm{SHH}$ signaling in the brain, producing tumors that resemble human $\mathrm{SHH}$ medulloblastoma in site, pathology, gene expression and cellular heterogeneity $[5,7,8]$. Studies of SHH-driven medulloblastomas in mice show that cellular heterogeneity contributes to tumor recurrence as individual cell types within tumors show different sensitivity or resistance to specific therapies and cells that survive treatment drive recurrence $[9,10]$. Tumor stem cells, marked by expression of OLIG2, are specifically resistant to cytotoxic treatments currently used in medulloblastoma therapy and initiate post-treatment recurrence [9]. Currently, $20 \%$ of patients with SHH subgroup medulloblastoma experience incurable recurrence despite optimal up-front therapy. 
Identifying ways to target therapy-resistant tumor stem cells may allow conventional treatment to be effective for more patients.

The cellular heterogeneity in SHH-driven medulloblastoma predominantly reflects the range of differentiation states assumed by normal cells over the course of cerebellar development; different types of medulloblastoma cells resemble undifferentiated stem cells, committed cerebellar granule neuron progenitors (CGNPs) or differentiated neurons [11]. However, medulloblastomas also contain tumor-derived cells with glial differentiation, and this divergence from the neural lineage of CGNPs provides indirect evidence that some medulloblastoma stem cells have glio-neuronal potency $[10,12]$. Malignant glia contributes to tumor growth by stimulating myeloid cells in the tumor microenvironment (TME) to secrete IGF1, which stimulates mTORC1 signaling in tumor cells through a paracrine loop [12].

AMP-activated Kinase (AMPK) is a multi-subunit complex that is a primary regulator of cellular energy homeostasis [13], and disrupting AMPK function may produce important anti-tumor effects [14]. The coordination of energy metabolism may be particularly important in $\mathrm{SHH}$ medulloblastomas which configure their energy metabolism to support malignant growth [15]. Examples of SHH-induced metabolic adaptations include HK2-dependent aerobic glycolysis [16, 17], lipogenesis [18], HIF1? stabilization[19] and mitochondrial fragmentation [20].

While AMPK is activated by the tumor suppressor LKB1, evidence suggests that AMPK may not also function as a tumor suppressor. Although inactivating LKB1 mutations have been found in diverse human cancers [21-23], mutations in AMPK subunits causing human cancer are unknown. Divergent roles of LKB1 and AMPK are 
demonstrated by the Kras ${ }^{G 12 D}$ mouse lung tumor model, in which $L k b 1$ deletion accelerates tumor growth while co-deletion of the two AMPK catalytic subunits Prkaa1 and Prkaa2 inhibits tumorigenesis. [24]. This divergence provides evidence that AMPK function is not limited to downstream modulation of LKB1. Moreover, diverse studies show that AMPK functions to increases metabolic adaptability in normal cells that may also be advantageous in cancer [25]. Experimental systems are needed to define the specific effects of AMPK in individual cancers.

HK2-dependent glycolysis plays an important role in maintaining tumor cells in undifferentiated states, as medulloblastomas that form in mice with brain-specific Hk2 deletion show increased terminal differentiation, resulting in slower tumor progression and markedly longer survival in the absence of anti-tumor treatment. Hk2-deleted medulloblastomas consistently show AMPK hyper-activation, implicating AMPK in regulating the balance of proliferation and differentiation. Importantly, AMPK can be inactivated in the mouse brain without producing discernable developmental defects or functional impairments [26], suggesting that therapies that disrupt AMPK in pediatric patients may effectively target medulloblastomas without harming normal brain development or maintenance.

Prior studies show that AMPK acts in medulloblastoma to support tumor growth, as deletion of Prkaa2, the gene encoding the AMPK22 catalytic domain slows tumor progression in mice engineered to develop medulloblastoma [27]. One AMPK-mediated mechanism that supports medulloblastoma progression is $\mathrm{SHH}$-induced phosphorylation of the zinc finger protein CNBP, which increases the translation of ornithine decarboxylase (ODC) and the production of polyamines [27, 28]. However, the 
mechanisms through which polyamine production permits tumor growth are unresolved, and other AMPK-dependent mechanisms may also affect medulloblastoma growth. Energy metabolism in medulloblastoma cells changes with differentiation state [16], and this variation suggest that specific types of tumor cells within medulloblastomas may depend on AMPK for different purposes.

To resolve AMPK function in different tumor cell subpopulations within medulloblastomas, we investigated the effect of AMPK catalytic domain ablation through Prkaa1 and Prkaa2 co-deletion in medulloblastomas that form in genetically engineered mice. We used scRNA-seq and immunohistochemistry to examine the impact of AMPK inactivation on different types of cells within medulloblastomas, and compared to Hk2-deleted medulloblastomas to probe the mechanistic role of altered metabolism. Our studies show that Prkaa1/Prkaa2 co-deletion, like Hk2 deletion, increased intra-tumoral differentiation. Notably, Prkaa1/Prkaa2 co-deletion reduced the populations of OLIG2-expressing stem cells that drive recurrence after conventional therapy. These studies provide new mechanistic insight into how metabolic specialization supports the diverse communities of tumor cells that promote recurrence after therapy and suggest a role for AMPK inhibition in targeting tumor stem cell populations that are typically refractory to conventional therapies. 


\section{Results}

\section{AMPK inactivation in medulloblastomas increase survival of tumor-bearing mice.}

To inactivate AMPK broadly in the CNS and then determine the effect on medulloblastoma, we generated medulloblastoma-prone mice with brain-specific Prkaa1/Prkaa2 co-deletion. We bred these mice by first crossing Gfap-Cre mice that express Cre recombinase in CNS stem cells during development with Prkaa1 ${ }^{\text {loxP/loxP } / P r k a a 2 ~}{ }^{\text {loxP/loxP }}$ mice that harbor loxP sites flanking the coding regions of Prkaa1 and Prkaa2 [26]. The resulting Gfap-Cre/Prkaa1/oxP/loxP/Prkaa2 loxPloxP (G$C r e^{A m p k}$ mice were born at Mendelian ratios and showed no deficits, consistent with our prior studies [26]. Next, we bred Prkaa1 $1^{\text {loxP/loxP/Prkaa2 loxP/loxP }}$ mice with the SmoM2 mouse line [29] to generate mice with Cre-conditional AMPK inactivation and Creconditional SHH-pathway hyperactivation (SmoM2 $\left.2^{A m p k}\right)$. We then crossed G-Cre ${ }^{A m p k}$ and SmoM2 $2^{A m p k}$ lines to produce G-Smo ${ }^{A m p k}$ mice that featured AMPK inactivation, and $\mathrm{SHH}$ hyperactivation throughout the brain.

Brain-wide SHH hyperactivation in Gfap-Cre/SmoM2 (G-Smo) mice is known to produce medulloblastoma with $100 \%$ penetrance by postnatal day 10 (P10) and no other brain tumors [30]. Similar to G-Smo mice, all G-Smo ${ }^{A m p k}$ mice developed medulloblastoma (Fig. 1A,B). In the absence of treatment, all G-Smo mice die of tumor progression by P22 [16, 31]. In contrast, untreated G-Smo ${ }^{A m p k}$ mice showed significantly longer survival times (Fig. 1C). These data show that AMPK inactivation in the G-Smo model does not prevent medulloblastoma formation, but impairs medulloblastoma progression, consistent with prior studies of isolated Prkaa2 deletion in $\mathrm{SHH}$ medulloblastomas driven by the $S m o A 1$ allele. 
A.
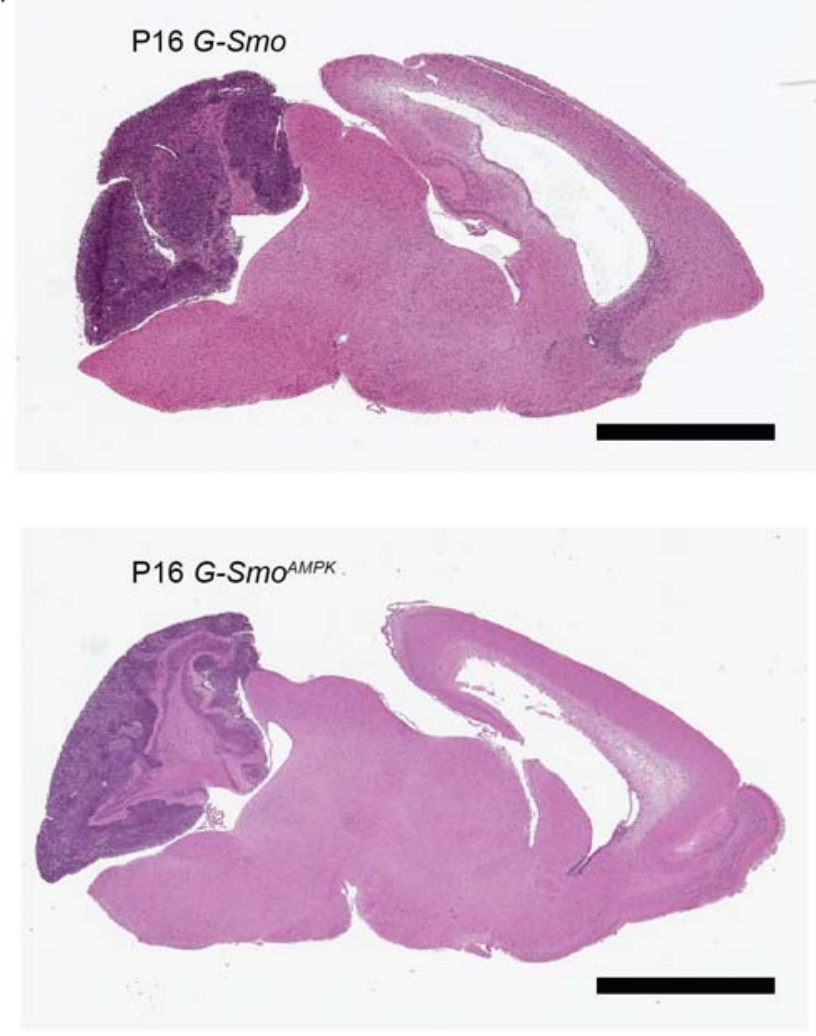

B.
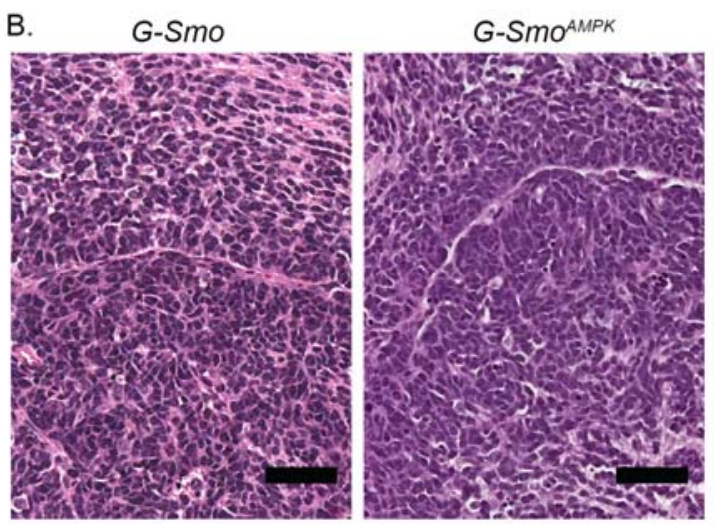

c.

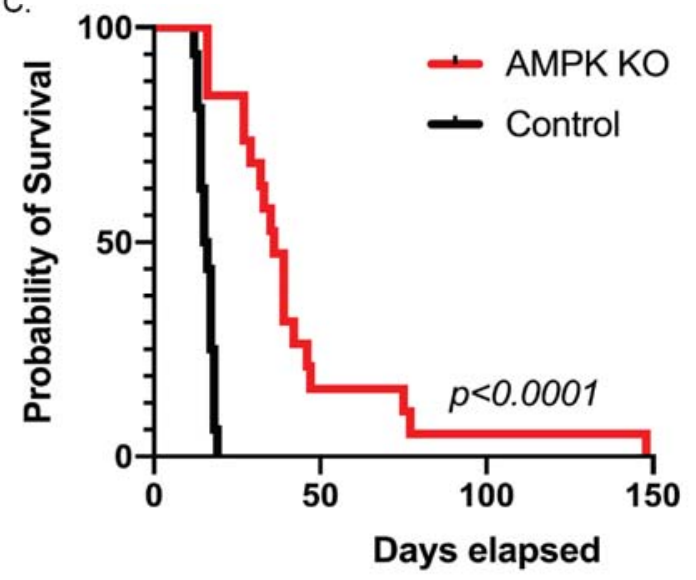

Figure 1. AMPK inactivation slows the progression of SHH-driven medulloblastoma. (A,B) Representative sagittal brain sections from G-Smo and G$S m o^{A M P K}$ mice show similar site of tumor formation and hypercellular tumor pathology. (C) Increased survival time in G-Smo ${ }^{A M P K}$ mice compared to G-Smo controls with intact AMPK. Bars $=3 \mathrm{~mm}$ in (A) and $50 \mu \mathrm{m}$ in (B). Survival times in (C) were compared by Log Rank test.

\section{AMPK inactivation alters medulloblastoma cellular heterogeneity and induces differentiation}

To determine if AMPK deletion differentially affects specific types of cells within medulloblastomas, we used gene expression analysis with single cell resolution (scRNA-seq) to compare tumors from 5 replicate P15 G-Smo ${ }^{A m p k}$ mice to 5 control medulloblastomas from replicate P15 G-Smo mice with WT Prkaa1 and Prkaa2 loci. We processed tumors using Drop-seq and generated cell-specific, bar-coded sequencing data, using our published methods $[10,31]$. We filtered putative cells, identified by 
bead-specific barcodes, to address the common problems of gene drop out, unintentional cell-cell multiplexing and premature cell lysis [32, 33]. 4,930 out of 6,743 putative G-Smo ${ }^{A m p k}$ cells, and 8699 out of 16489 G-Smo cells met criteria and were included in the analysis. To compare the two genotypes at similar sequencing depths, we randomly down-sampled the G-Smo transcript counts to $46.5 \%$ of the original depth [34].

We used the Harmony algorithm to integrate the scRNA-seq data from GSmo ${ }^{A m p k}$ and G-Smo tumors in a single analysis, generating a 2-dimensional UMAP projection in which individual cells were clustered with cells of similar gene expression. As in prior studies, this analysis defined both discrete clusters and a group of clusters with shared borders (Fig 2A). We determined the biological relevance of the clustering by generating cluster-specific differential gene expression profiles (Supplementary Data 1). For each detected gene, we compared the expression by cells within the cluster to the expression by all cells outside the cluster. We then identified the cell type of each cluster by its gene expression profile.

As in our prior studies, these methods identified the discrete clusters as different types of stromal cells typical of brain tissue including: astrocytes, oligodendrocytes, ependymal cells, myeloid cells, endothelial cells and fibroblasts (Table 1; Fig. 2A). Expression of CGNP markers Barhl1 and NeuroD1 identified the 8 clusters in the multicluster complex as tumor cells (Fig. 2B). Cluster-specific gene expression analysis showed these clusters to represent a range of states that paralleled CGNP development, from proliferative, undifferentiated cells to non-proliferative cells at different stages of neural differentiation as described below and in Table 1 . The full set 
of gene expression data with single cell resolution from G-Smo ${ }^{A M P K}$ and G-Smo control tumors can be viewed at: https://malawsky.shinyapps.io/Gerhson_AMPK_analysis.

To parse the multi-cluster complex of tumor cells, we designated tumor cell clusters as proliferative based by expression of proliferation markers Mki67 and Cyclin genes and SHH transcription factor Gli1 (Fig. 2B). We further characterized proliferative clusters as quiescent, cycling or M-phase enriched based on expression of S/G2/M marker Top2a [35] and mitotic marker Ccnb1 [36](Fig. 2B). The non-proliferative clusters showed successive expression of early to late differentiation markers NeuroD1, Cntn2, and Grin2b (Table 1; Fig 2B) with terminally differentiated neurons as the most differentiated cell type within this group [10, 31]. Using these markers, we classified the tumor cell clusters as $(A)$ proliferative but quiescent, $(B)$ proliferative and cycling, or $(C)$ differentiating (Fig 2C,D; Table 1).

\begin{tabular}{|l|l|l|l|}
\hline Cluster & Cell type designation & distinctive markers & Tumor cell classification \\
\hline 0 & tumor cells & Barhl1 & A- proliferative, quiescent \\
\hline 1 & early differentiating tumor cells & Tubb3, Stmn2 & C- differentiating \\
\hline 2 & proliferating tumor cells & Top2a, Dek & B- proliferative, cycling \\
\hline 3 & tumor cells & Gas5, Rps20 & A- proliferative, quiescent \\
\hline 4 & differentiating tumor cells & Stmn2, Celf4, Nrxn1 & C- differentiating \\
\hline 5 & mitotic tumor cells & Cenpa, Ube2c & B- proliferative, cycling \\
\hline 6 & late differentiating tumor cells & Cntn2, Gria2, Apc & C- differentiating \\
\hline 7 & proliferating tumor cells & Top2a, Mki67 & B- proliferative, cycling \\
\hline 8 & proliferating tumor cells & Top2a, Mki67, Ube2c & B- proliferative, cycling \\
\hline 9 & unresolved & Mt1, Mt2 & stromal \\
\hline 10 & immature oligodendrocytes & Olig1/2, Pdgfra & stromal \\
\hline 11 & myeloid cells & C1qc/a, Tyrobp & stromal \\
\hline 12 & myelinating oligodendrocytes & Plp1, Mbp & stromal \\
\hline 13 & endothelial cells & Cldn5 & Stromal \\
\hline 14 & fibroblasts & Dcn & stromal \\
\hline 15 & astrocytes & Aqp4, Slc1a3 (aka Glast) & stromal \\
\hline
\end{tabular}




\section{Table 1. Identification of clusters as specific types of tumor and stromal cells}

We disaggregated the cells by genotype (Fig. 2E) to compare patterns of the populations and gene expression patterns of each cell types in G-Smo ${ }^{A m p k}$ and G-Smo control tumors. We compared the distribution of tumor cells across the differentiation spectrum by determining the number of cells from each replicate animal in Set $A$, B or C, normalized to the total number of cells from that animal (Fig. 2F). The distribution of tumor cells across the three sets was significantly different in G-Smo ${ }^{\text {Ampk }}$ tumors $(p=0.034 ;$ ANOVA), with differentiated cells (Set $C)$ significantly increased $(p=0.01$; Student's t-test).

Similarly, we compared the populations of each stromal cluster in G-Smo ${ }^{A m p k}$ and G-Smo control tumors (Fig. 3G). We did not detect significant differences in most stromal populations, including astrocytes and myelinating oligodendrocytes which were both within the GFAP lineage and thus subject to Prkaa1/2 deletion. However, immature oligodendrocytes of Cluster 10, which were also subject to Prkaa1/2 deletion, were 3fold less numerous in G-Smo ${ }^{\text {Ampk }}$ tumors ( $p=0.02$; Student's t-test). Additionally, G$S m o^{A m p k}$ tumors showed a 3-fold increase in endothelial cells, which were not within the GFAP lineage and thus not Prkaa1/2-deleted ( $\mathrm{p}=0.02$; Student's t-test). This increase in endothelial cells likely indicates increased vascularization. AMPK inactivation thus increased differentiation in both the tumor and oligodendrocyte lineages and also produced a non-cell autonomous increase in endothelial cells. 


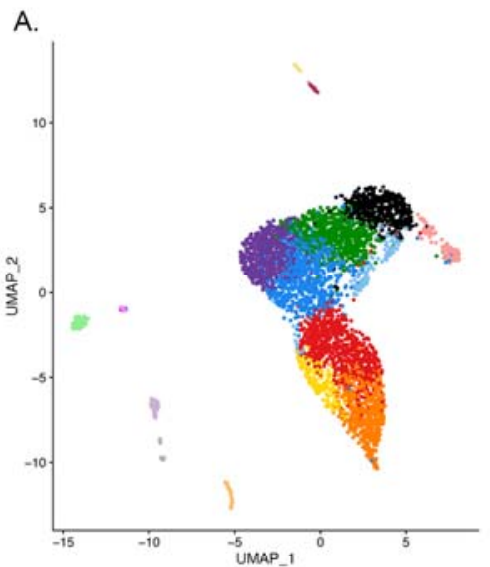

D.

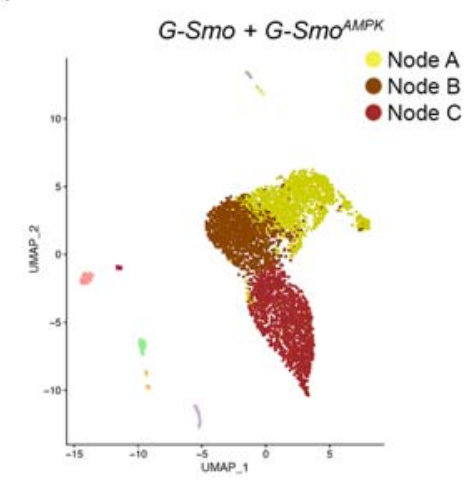

B.

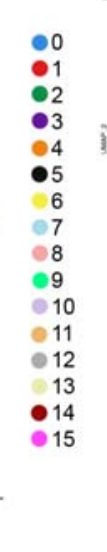

E.

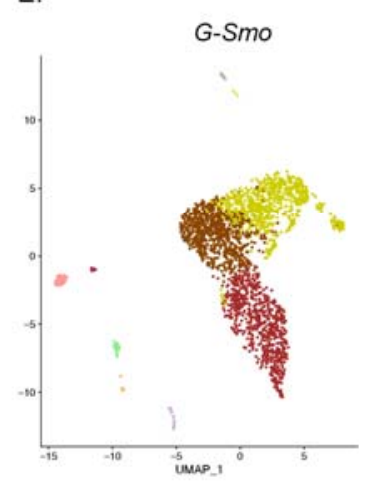

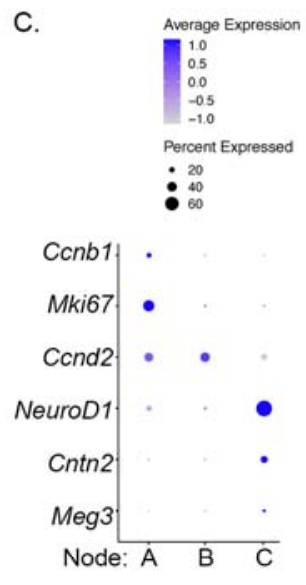

G-SmoAMPK
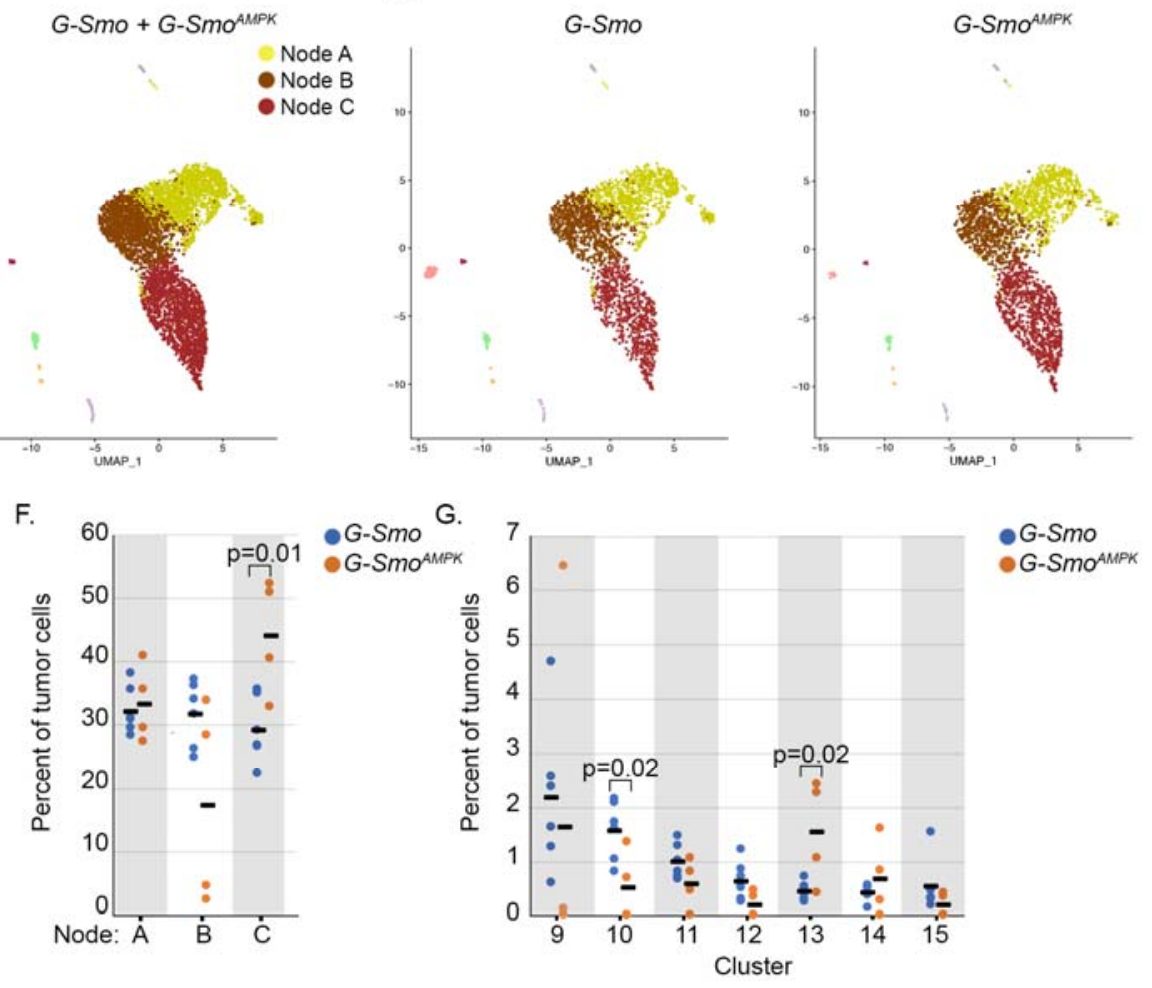

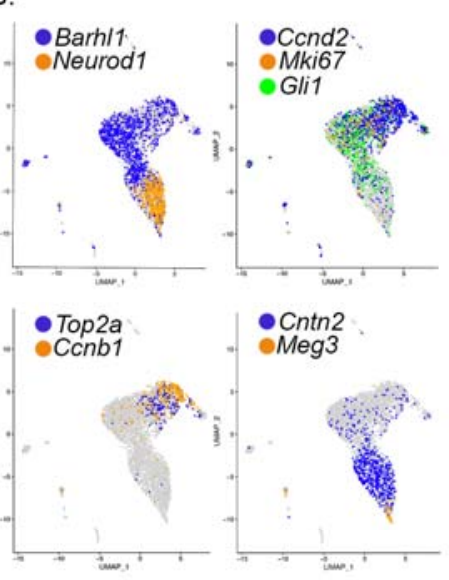

- Barh

-G-Smo

Figure 2. AMPK inactivation alters cellular heterogeneity in SHH driven medulloblastomas. (A) UMAP plot of all cells from G-Smo and G-Smo ${ }^{A M P K}$ tumors, color-coded by cluster. Cells are localized according to their proximity in PCA space. (B) Feature plots showing expression of indicated proliferation or differentiation markers, color coded over the UMAP shown in (A). (C) Dot plot of indicated markers that division of tumor cells into Nodes A-C separates them by proliferation and differentiation states. (D) Nodes A-C projected onto the UMAP from (A). (E) UMAP plots deconvoluted by genotype, with Nodes $A-C$ color coded. $(F, G)$ Comparison of populations of $(F)$ Nodes A-C or (G) each stromal cluster in G-Smo and G-Smo ${ }^{A M P K}$ tumors. Dots represent values for individual replicates and horizontal bars indicate the means. Student's t-test was used to make pairwise comparison. 


\section{AMPK-inactivated medulloblastomas show reduced OLIG2+ stem cells}

Tumor stem cells play an essential role in medulloblastoma progression. Considering the extended survival time and increased differentiation in AMPKinactivated medulloblastomas, we determined if stem cell populations were reduced. We compared the populations of cells that expressed the stem cell markers markers Hes1, Olig2, Sox2, Nes and Vim in G-Smo versus G-Smo ${ }^{A M P K}$ tumors (Fig. 3A). In the tumor cell clusters of Nodes A-C, Olig2+ and Vim+ cells were significantly reduced in GSmo ${ }^{A M P K}$ medulloblastomas, while Sox2+ cells and Hes1+ cells showed trends toward reduced numbers that were not statistically significant. To further compare stem cell populations, we quantified cells in medulloblastomas that expressed OLIG2 protein, and then subtracted OLIG2+ cells that also expressed the oligodendrocyte marker SOX10, both detected by immunohistochemistry (Fig. 3C). The number of OLIG2+/SOX10- cells were markedly reduced in G-Smo ${ }^{A m p k}$ tumors, consistent with the differences in Olig2+ populations in Nodes A-C in the scRNA-seq data (Fig. 3C). These scRNA-seq and immunohistochemistry studies consistently demonstrate a profound decrease in stemlike populations in AMPK-inactivated medulloblastomas.

\section{AMPK-inactivation disrupts tumor to glia trans-differentiation}

Lineage tracing in mouse models of $\mathrm{SHH}$ medulloblastoma show that tumor cells trans-differentiate to generate tumor-derived glial populations [10, 12], and that these malignant glia play a supportive role in tumor growth [12]. Glial trans-differentiation requires stem-like pluripotency, and in light of the reduced stem-like population in G- 
$S m o^{A m p k}$ tumors, we compared glial trans-differentiation in G-Smo ${ }^{A m p k}$ and G-Smo control tumors.

We traced lineage in the scRNA-seq data using the 3' Yfp sequence in the Creconditional SmoM2 transgene. We previously used this method to show that subsets of astrocytes and oligodendrocytes derive from the tumor lineage in medulloblastomas that form in Math1-Cre/SmoM2 mice [10]. In the G-Smo control tumors, we found SmoM2Yfp expression in glial cells (Fig. 3D), which was expected since Gfap-Cre activates SmoM2 in glio-neuronal stem cells of the developing brain that generate both glial and neuronal progeny. However, not all glia were SmoM2-Yfp+, indicating that SmoM2 activation was not uniform in these populations, and this variation allowed us to quantify the glial cells of tumor lineage. G-Smo ${ }^{A m p k}$ tumors showed significantly smaller SmoM2Yfp+ glial populations, indicating fewer tumor-derived glia (Fig. 3D, p-value 0.024). The decreased glial trans-differentiation provides functional evidence that stem cells were reduced in G-Smo ${ }^{\text {Ampk }}$ tumors. 

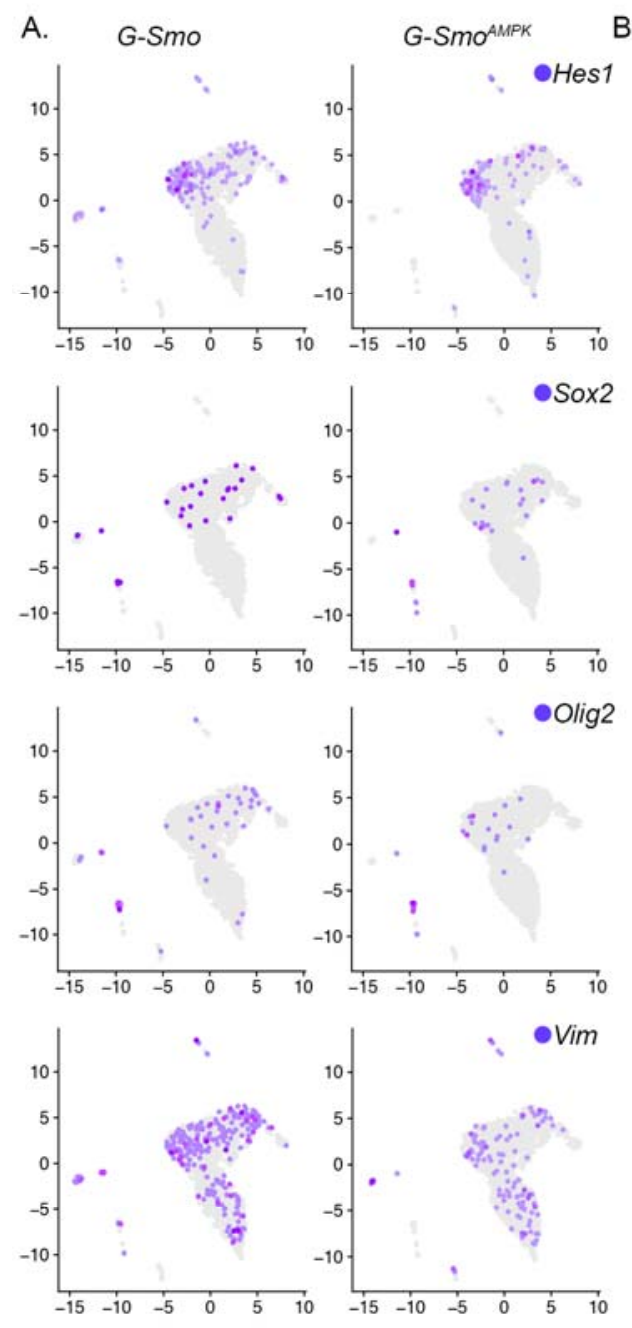

C.

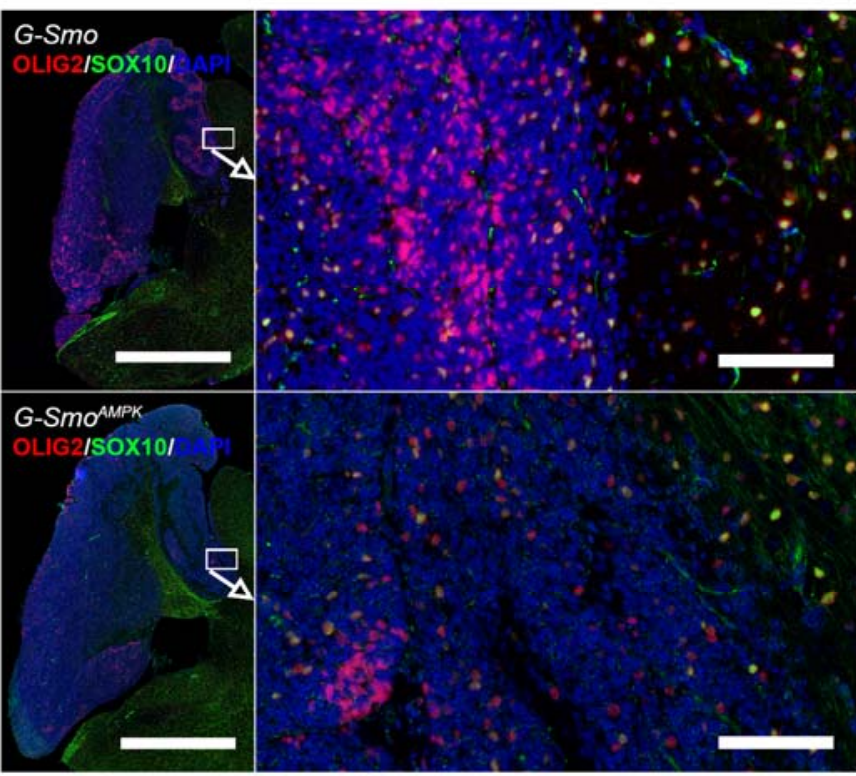

D.
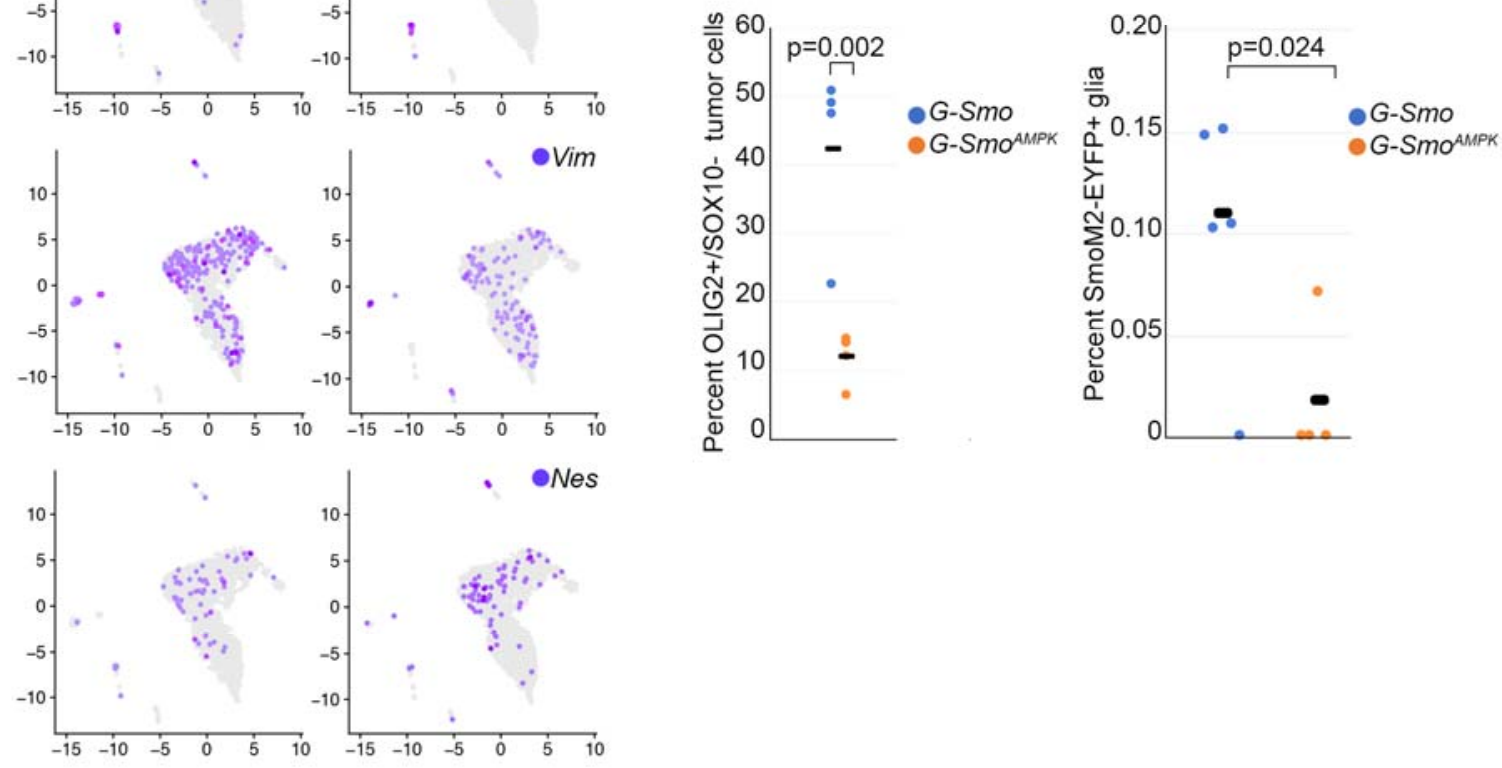

Figure 3. Decreased stem cell populations in G-Smo ${ }^{A M P K}$ tumors. (A) Expression of indicated stem cell markers in the indicated genotypes, projected onto the disaggregated UMAPs. Hes1, Sox2, Olig2 and Vim were significantly lower in G$S m 0^{A M P K}$ tumors, while Nes was not significantly different. (B) Representative OLIG2/SOX10 IHC in sagittal sections of tumors of indicated genotype. (C) Quantification of OLIG2/SOX10 IHC as in (B) in replicate samples of each genotype. (D) 
Quantification of SmoM2-YFP+ glial cells in the indicated genotypes. In (C) and (D), pvalue was determined student's t-test.

\section{Decreased mTORC1 activation in AMPK-inactivated medulloblastomas}

To gain insight into the processes that reduce tumor progression and stem cell populations in G-Smo ${ }^{A m p k}$ medulloblastomas, we analyzed the transcriptomic effect of AMPK-inactivation on each type of tumor cell. We determined for each cluster of tumor cells the set of genes differentially expressed by cells from G-Smo ${ }^{\text {Ampk }}$ mice versus GSmo controls. The resulting cluster-specific differential gene list showed a highly consistent pattern of down-regulated genes. Of 36 different genes significantly downregulated in any of the 9 tumor cell clusters, 14 were down-regulated in at least 5 of 9 clusters, and 11 were down-regulated in at least 7 of 9 clusters (Fig. $4 A, B ; p<10^{-15}$ by extended hypergeometric test [37]). These 14 genes encoded multiple ribosomal proteins and the translation elongation factor EEF1B2, suggesting an effect of protein translation. Similarly, GO analyses of this set identified translation as the primary process statistically implicated (corrected $\mathrm{p}<4 \times 10^{-23}$ ). In view of the consistent downregulation of translation-related genes in multiple types of tumor cells, we analyzed whether mTORC1 signaling was altered in G-Smo ${ }^{A m p k}$ tumors.

We assessed mTORC1 activation state by analyzing the expression of phosphorylated 4EBP1 (p4EBP1), detected by immunohistochemistry. We quantified p4EBP1+ cells in tissue sections of G G-Smo ${ }^{A m p k}$ and control tumors (Fig. 4C). GSmo ${ }^{\text {Ampk }}$ tumors showed significantly fewer p4EBP1+ cells, and an F-score that integrated both the fraction of positive cells and the staining intensity was similarly decreased (Fig. 4D). Together the patterns of gene expression and p4EBP1 studies show reduced mTORC1 activation in G-Smo ${ }^{\text {Ampk }}$ tumors compared to G-Smo control 
tumors. AMPK activation is known to inhibit mTORC1 [38-40], and the finding of reduced mTORC1 activation $\mathrm{G}-S m 0^{A m p k}$ tumors suggests a homeostatic response to chronic AMPK inactivation.

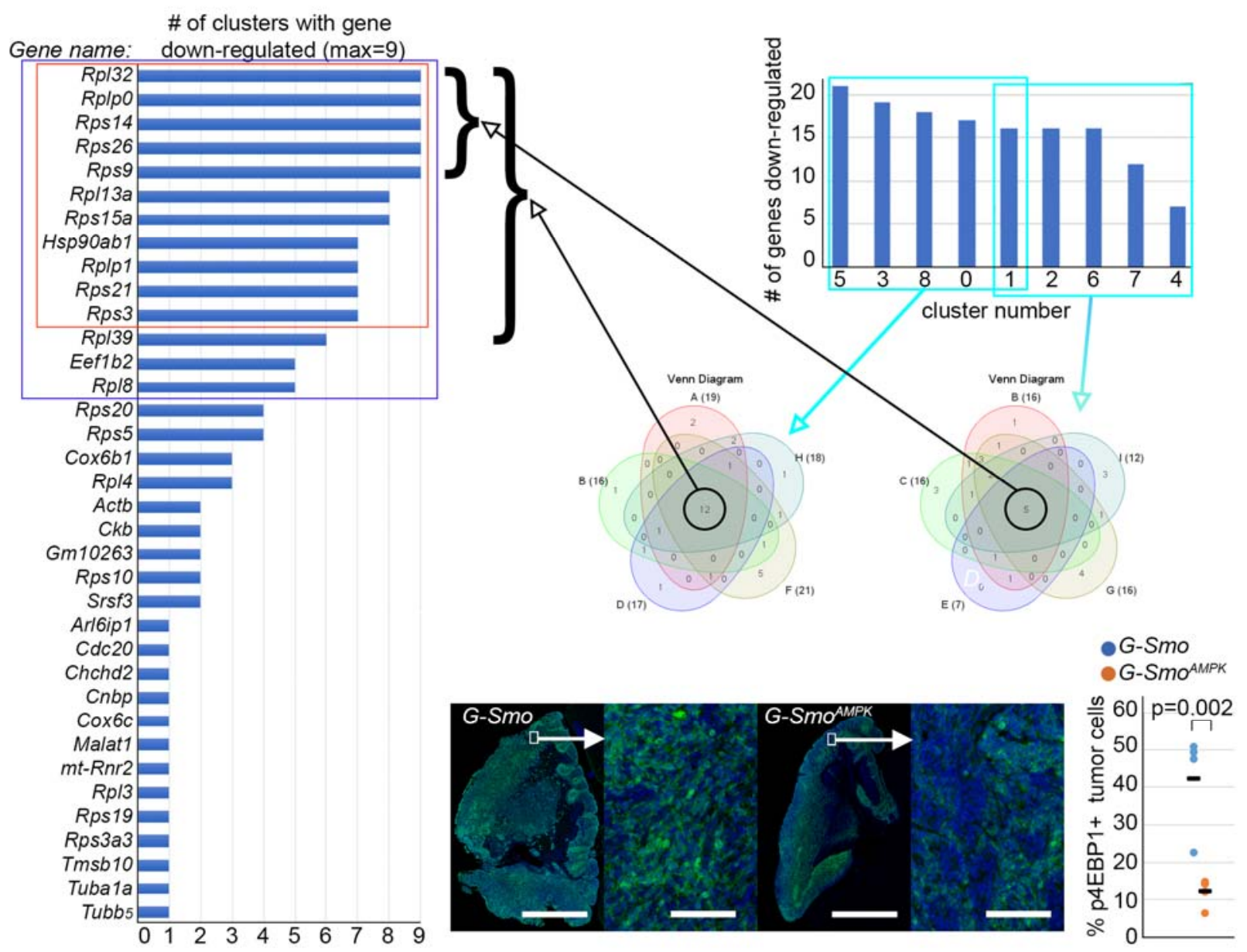

Figure 4. Decreased mTORC1 activity in G-Smo ${ }^{A m p k}$ tumors. (A) Genes that are down-regulated in 1 or more clusters, ranked by number of clusters with differential expression. Red box marks the 11 genes down-regulated in $7 / 9$ clusters. Blue box marks the 14 genes down regulated in 5/9 clusters. (B) Venn diagrams of the genes down-regulated in the $5 / 9$ clusters with the highest number of differentially expressed genes, or in the 5/9 clusters with the fewest differentially expressed genes. Cluster 1 is included in both Venn diagrams as a point of consistency, as depicted in the graph. These Venn diagrams depict sets of 12 or 5 genes down-regulated in all included clusters and brackets show the indicated sets of genes commonly down-regulated genes in the list from (A). (C) Representative p4EBP1 IHC in sagittal sections of tumors of indicated genotype. (D) Quantification of p4EBP1 IHC as in (C) in replicate samples of each genotype. 


\section{Altered glycolytic gene expression in AMPK-inactivated medulloblastomas}

We previously found that HK2-dependent aerobic glycolysis maintains medulloblastoma undifferentiated populations within SHH-driven medulloblastomas. In these prior studies, we crossed G-Smo mice with mice that harbor conditional Hk2 deletion $\left(H k 2^{\text {loxP/loxP }}\right)$ to produce the genotype Gfap-Cre/SmoM2/Hk2 ${ }^{\text {loxP/loxP }}\left(G-S m o^{H k 2}\right.$ ) and found that the medulloblastoma that form in ${\mathrm{G}-S m o^{H k 2}}^{H i c e}$ showed markedly increased differentiation and event-free survival, and also increased AMPK activation [16]. The correlated increases in differentiation and AMPK activation in Hk2-deleted tumors suggested that AMPK activation might induce differentiation. Our current finding of increased differentiation in G-Smo ${ }^{\text {Ampk }}$ tumors, however, demonstrates that differentiation can proceed in the absence of AMPK activity.

To define more clearly the interrelation of AMPK and HK2, we bred Hk2 ${ }^{\text {loxP/loxP }}$ mice with G-Smo ${ }^{\text {Ampk }}$ mice to generate Gfap-Cre/Prkaa1/oxP/loxP/Prkaa2 IoxP/loxP/Hk2 IoxP/loxP $\left(G-S m o^{A m p k / H k 2}\right)$ mice with brain-wide AMPK inactivation, Hk2 deletion and SmoM2 expression. These G-Smo ${ }^{A m p k / H k 2}$ mice developed medulloblastomas with $100 \%$ frequency, and the tumors showed increased differentiation, resembling the previously described differentiated medulloblastomas in G-Smo ${ }^{H k 2}$ mice (Fig. 5A) [16]. These codeletion studies show that the increased differentiation phenotype caused by $H$ k2 deletion does not require AMPK activity. Thus, AMPK does not operate downstream of HK2-dependent glycolysis to regulate differentiation. In light of these findings, we 
examined the alternative possibility that AMPK may operate upstream to induce HK2dependent aerobic glycolysis.
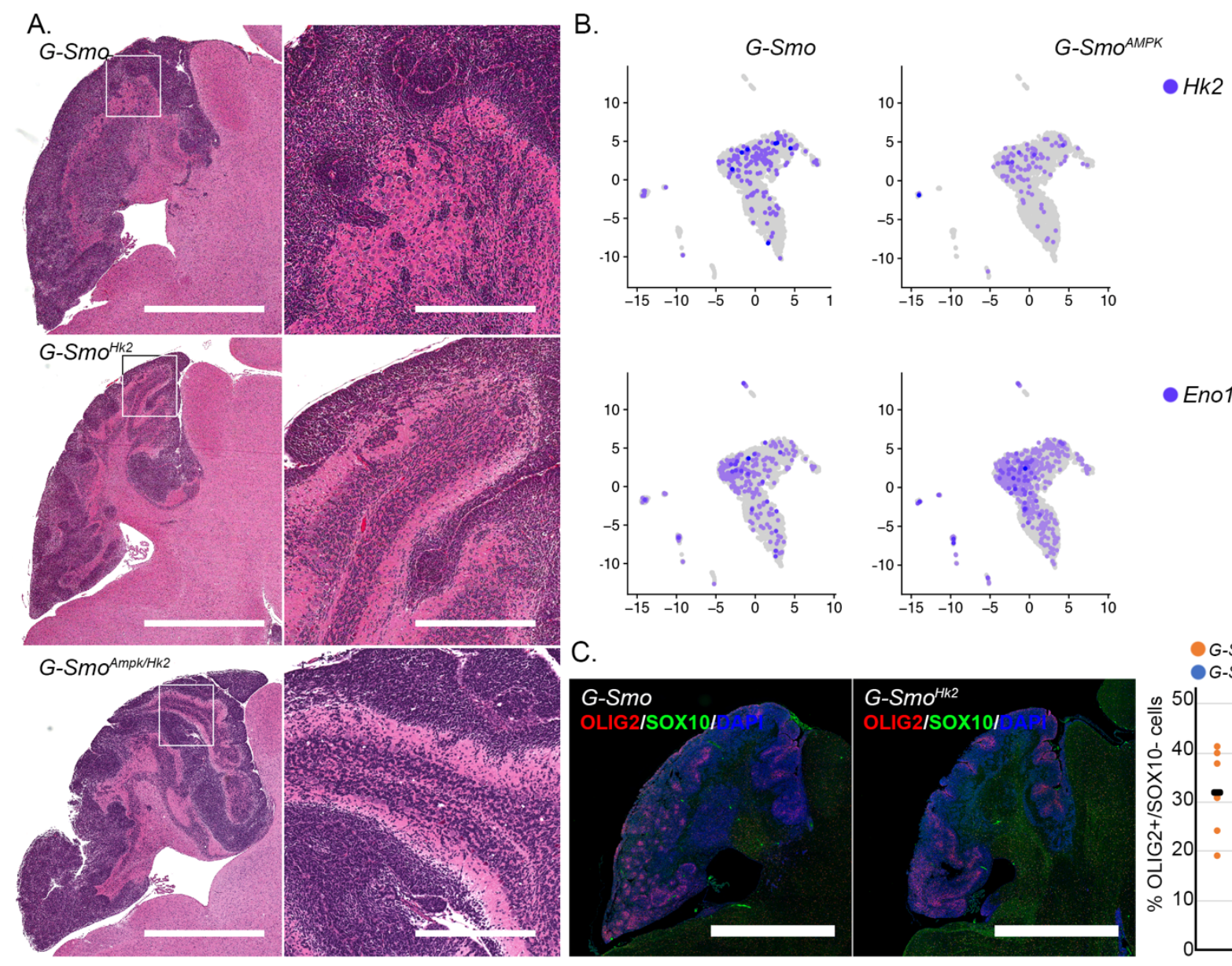

Eno1

C.
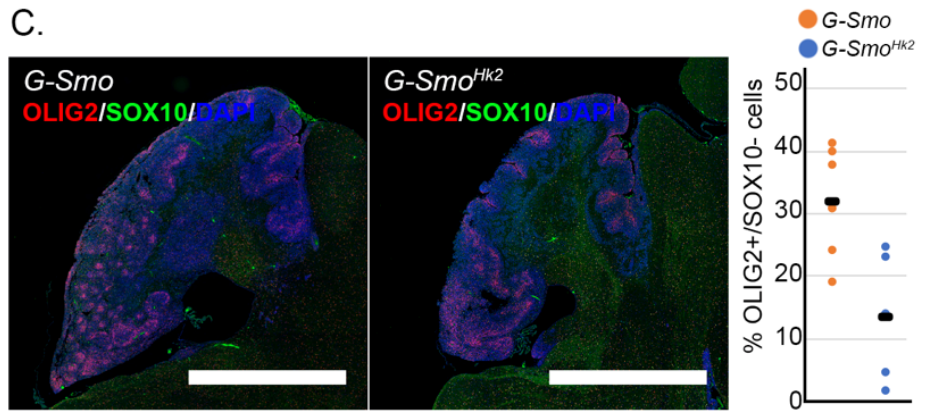

Figure 5. Defining the interaction of AMPK and HK2. (A) Representative H\&E sections compare G-Smo control tumors to tumors with deletion of Hk2 or co-deletion of Hk2, Prkaa1 and Prkaa2. Increased differentiation in Hk2-deleted tumors is not rescued by co-deletion of Prkaa1 and Prkaa2. (B) scRNA-seq data showing decreased Hk2 and increased Eno1 in M-Smo ${ }^{A M P K}$ tumors. (C) Representative OLIG2 immunofluorescence, comparing G-Smo and Hk2-deleted medulloblastomas, with quantification of replicate samples. $P$ value determined by Student's t-test.

We compared the number of cells expressing each gene in the glycolytic pathway in G-Smo ${ }^{A m p k}$ medulloblastomas compared to G-Smo control tumors and found that Hk2 and Eno1 were differentially expressed in AMPK-deleted tumors. Hk2+ cells 
were significantly reduced in G-Smo ${ }^{A m p k}$ tumor cells (Fig. 5B), consistent with the hypothesis that AMPK activity modulates $\mathrm{HK} 2$ expression in $\mathrm{SHH}$-driven medulloblastoma, as seen in prior studies of normal in muscle cells [41]. In contrast, cells expressing Eno1, which acts at the end of the glycolytic pathway, were increased in G-Smo ${ }^{A m p k}$ tumors (Fig. 5B). AMPK inactivation thus induced specific changes in glycolytic gene expression.

Similar stem cell changes in AMPK-inactivated and Hk2-deleted medulloblastomas

To determine if alterations in glycolysis may be sufficient to recapitulate aspects of the AMPK-inactivation phenotype, we analyzed OLIG2+ populations in Hk2-deleted medulloblastomas. Comparing G-Smo/Hk2 floxed mice to G-Smo controls we found that Hk2-deletion was sufficient to reduce the fraction of OLIG2+ tumor cells (Fig. 5C). Reduced tumor stem cell populations was thus a common feature of AMPK-inactivated and Hk2-deleted medulloblastomas, suggesting that both genes operate in the same pathway to maintain tumor stem cell populations.

\section{Discussion}

Medulloblastomas are highly proliferative tumors that configure energy metabolism to support malignant growth through enhanced aerobic glycolysis $[16,17]$ and lipogenesis [18, 42]. AMPK is an intracellular energy sensor that coordinates anabolic and catabolic processes with nutrient availability and may crucially regulate tumor metabolism in ways that might be predicted either to support or to inhibit tumor 
growth. In SHH signaling, AMPK has been shown to interact directly with GLI1 to suppress SHH activity in medulloblastoma [43, 44]. In contrast to the anti-tumor effects of AMPK predicted by its interactions with GLI1, however, a prior study of $\mathrm{SHH}$ medulloblastoma showed that deletion of AMPK subunit Prkaa2 slowed tumor progression in a primary mouse model [27]. Understanding the mechanisms through which AMPK inactivation reduces medulloblastoma growth may allow the design of targeted therapies that exploit the role of AMPK in SHH medulloblastoma (and potentially in other cancers).

Our data show that AMPK inactivation slowed medulloblastoma growth and altered tumor cell heterogeneity by disproportionately impacting tumor stem cells. We inactivated AMPK by conditionally deleting both catalytic subunits, Prkaa1 and Prkaa2, in mice carrying Gfap-Cre and SmoM2 alleles, generating G-Smo ${ }^{\text {Ampk }}$ mice with AMPK inactivation and $\mathrm{SHH}$ hyperactivation throughout the brain. In these mice, AMPKinactivated medulloblastomas formed with $100 \%$ penetrance but progressed more slowly than medulloblastomas in controls with $\mathrm{SHH}$ hyperactivation but intact AMPK. Like control tumors, AMPK-inactivated tumors comprised medulloblastoma cells with a range of differentiation states. However, AMPK-inactivated medulloblastomas showed a shift in cell populations, with increased differentiated cells compared to controls and specifically reduced populations of OLIG2+ stem cells and malignant glia.

Along with changes in cellular composition, AMPK inactivation altered gene expression in multiple cell types. G-Smo ${ }^{A m p k}$ medulloblastomas showed altered expression of glycolytic genes, with decreased Hk2 and increased Eno1. By impeding the entry of glucose into the glycolysis through HK2 and enhancing the exit of glycolytic 
intermediates as phosphoenolpyruvate through ENO1, AMPK inactivation reduced the potential for diversion of glycolytic intermediates for other purposes. AMPK inactivation also decreased the expression of multiple genes related to protein translation in tumor cells across the differentiation spectrum, indicating reduced mTORC1 activity. This global decrease in mTORC1 signaling was unexpected, as acute AMPK activation inhibits mTOR $[45,46]$. We speculate that $G-S m o^{A m p k}$ tumor cells reduce mTORC1 in a homeostatic response to AMPK inactivation.

We noted specific changes in the stromal populations in AMPK-inactivated tumors with important implications. Within the stromal populations subject to deletion of Prkaa1 and Prkaa2, the less mature oligodendrocyte subset was specifically depleted in G-Smo ${ }^{\text {Ampk }}$ medulloblastomas. These cells, like tumor stem cells, express OLIG2, and their decreased numbers suggests an interaction between AMPK and OLIG2 may crucially modulate OLIG2 function. Within the stromal populations that were not subject to Prkaa1 and Prkaa2 co-deletion, the myeloid subset was similarly reduced in G$S m o^{A m p k}$ medulloblastomas. As these cells were not within the GFAP-lineage, their reduced population is necessarily a non-cell autonomous effect that indicates a change in intercellular signaling.

By reducing stem cell self-renewal, glial trans-differentiation, HK2-dependent aerobic glycolysis and mTORC1 activation, AMPK inactivation altered multiple processes important for tumor progression. Each of these processes has been tested in isolation in previous studies. The maintenance of OLIG2+ stem cell pools has been shown to promote both medulloblastoma progression and recurrence after cytotoxic therapy [9]. HK2-dependent aerobic glycolysis is similarly required for tumor 
progression [16]. Paracrine signaling between malignant glia and myeloid cells within medulloblastomas also promotes tumor growth [12]. The activation of mTORC1 is similarly essential for tumor growth, and mTORC1 inhibition slows progression [47]. All of these processes are likely to be interrelated, and to contribute to the anti-tumor effect of AMPK inactivation. AMPK inactivation thus sets in motion a complex set of processes that result in the observed shift from multipotent stem cells to more differentiated tumor cells, with the net effect of slowing tumor growth.

To define causal relationships between processes that we found to be altered in AMPK-inactivated tumors, we compared the phenotypes of AMPK-inactivated and Hk2deleted tumors. Both Hk2 deletion and Prkaa1/Prkaa2 co-deletion slow tumor growth and extend event-free survival. Importantly, Hk2 deletion impaired stem cell maintenance and increased differentiation, reproducing the altered cellular heterogeneity of the Prkaa1/Prkaa2 co-deleted tumors. These genetic data provide evidence for a linear pathway in which AMPK and HK2 both operate in the same direction to maintain undifferentiated, pluripotent populations within medulloblastomas.

The effect of AMPK inactivation on OLIG2+ stem cells identifies a vulnerability that may be exploited therapeutically for clinical benefit. Tumor stem cells are intrinsically resistant to cytotoxic therapy and drive recurrence. Ways to target these stem cells are urgently needed. Our data show that chronic blockade of AMPK activity disproportionately affects these stem cells, providing a therapeutic approach that may combine well with other medulloblastoma treatments, potentially increasing efficacy without producing overlapping toxicity. Follow up studies are needed to test pharmacologic AMPK inhibition in combination with current, cytotoxic therapy. As 
genetic deletion studies demonstrate that AMPK inactivation can suppress tumor growth, and AMPK deletion in model organisms does not have clear deleterious effects [26], AMPK inhibition may emerge as an important new avenue for cancer therapy.

\section{Methods}

Mice

We crossed SmoM2 mice ( Jackson Labs, stock \# 005130) with GFAP-Cre mice (Jackson Labs, stock \# 004600), to generate G-Smo mice. We crossed Prkaa1 1oxP/loxP/Prkaa2 loxP/loxP mice with GFAP-Cre mice to generate GfapCre/Prkaa1 ${ }^{\text {loxP/loxP } / \text { Prkaa2 loxP/loxP }}$ (G-Cre ${ }^{A m p k}$ ) mice. We then crossed G-Cre ${ }^{A m p k}$ mice with SmoM2 mice generate G-Smo ${ }^{A M P K}$ mice with Prkaa1/2-deleted medulloblastomas. All mice were of species Mus musculus and crossed into the C57BL/6 background through at least five generations. All animal studies were carried out with the approval of the University of North Carolina Institutional Animal Care and Use Committee under protocols (19-098 and 21-011).

\section{Histology and immunohistochemistry}

Mouse brains were processed, immunostained and quantitatively analyzed as previously described [10, 48, 49]. Primary antibodies used were: OLIG2 diluted 1:100 (Cell Marque, \# 387R-14), SOX10 diluted 1:200 (Cell Signaling Technology, \#7833S). Stained images were counterstained with DAPI, digitally imaged using an Aperio Scan Scope XT (Aperio) and subjected to automated cell counting using Tissue Studio (Definiens). 


\section{Tissue Preparation for Drop-seq}

Mice were euthanized by decapitation under isoflurane anesthesia. The brain was divided along the sagittal midline and one half was processed for histology and a sample of tumor was dissected from the other half and processed for Drop-seq analysis.

This sample was dissociated using the Papain Dissociation System (Worthington Biochemical) following the protocol used in previous studies ${ }^{[10,50]}$. Briefly, tumor samples were incubated in papain at $37 \square^{\circ} \mathrm{C}$ for $15 \mathrm{~min}$, then triturated and the suspended cells were spun through a density gradient of ovomucoid inhibitor.

We resuspended pelleted cells in $1 \mathrm{~mL}$ HBSS with $6 \mathrm{~g} / \mathrm{L}$ glucose and diluted in PBS-BSA solution to a concentration of $95-110$ cells/ $\mu \mathrm{L}$. Barcoded Seq B Drop-seq beads (ChemGenes) were diluted in Drop-seq lysis buffer to a concentration between 95-110 beads $/ \mu \mathrm{L}$. Tumor cells were co-encapsulated with barcoded beads using FlowJEM brand PDMS devices as previously described [10]. All cells were processed within one hour of tissue dissociation. Droplet breakage and library preparation steps followed Drop-seq protocol V3.1 [51]. After PCR, amplified cDNA was subjected Ampure XP cleanup at $0.6 \mathrm{x}$ and $1 \mathrm{x}$ ratios to eliminate residual $\mathrm{PCR}$ primers and debris. found by the bioanalyzer electropherogram. 1. If PCR failed to generate adequate cDNA, the PCR was repeated with the 3rd round increased from 11 to 13 cycles.

For QC purposes, library pools consisting of the tagmented cDNA from 2,000 beads/run were prepared and sequenced to low depth ( $2.5 \mathrm{M}$ reads/2K beads). We used the resulting data to assess library efficiency, including total read losses to PolyA 
regions, nonsense barcodes and adapter sequences as well as the quality and number of the transcriptomes captured. Passable runs contained $40-60 \%$ of reads associated with the top $80-100$ barcodes found in 2,000 beads.

Drop-seq runs passing QC were then prepared for high-depth sequencing on an Illumina Novaseq S2 flow cell.

\section{Processing of scRNA-seq data}

Data analysis was performed using the Seurat R package version 3.1.1 [52]. Data were subjected to several filtering steps. First, only genes that were detected in at least 30 cells were considered, to prevent misaligned reads appearing as rare transcripts in the data. Cells were then filtered using specific QC criteria to limit the analysis to cells with transcriptomes that were well-characterized and not apoptotic.

We noted that G-Smo cells were sequenced at a greater depth than G-Smo ${ }^{A M P K}$ cells which can introduce unwanted batch effects into the analysis. Consistent with best practices [34], we downsampled the G-Smo cells to $46.5 \%$ of their original depth so as to achieve similar sequencing depth between G-Smo and G-Smo ${ }^{A M P K}$ cells prior to further filtering.

We filtered out putative cells with fewer than 500 detected RNA molecules (nCount) or 200 different genes (nFeature), as likely to represent ambient RNA. We filtered out putative cells with greater than 4 standard deviations above the median nCount or nFeature as likely to be doublets, improperly merged barcodes, or 
sequencing artifacts. We also filtered out putative cells with more than $10 \%$ mitochondrial transcripts which we suspected to be dying cells

scRNA-seq Data normalization, clustering, differential gene expression, and visualization

We normalized the data was normalized using the SCTransform as implemented in Seurat, then selected the top 3,000 most highly variable genes. We performed PCA on these highly variable genes using the RunPCA function. The number of PCs to be used in downstream analysis was chosen based on the elbow plot as implemented by Seurat. We then used the FindNeighbors and FindClusters functions to identify cell clusters in the data.

To identify differential genes between clusters of cells, we used Wilcoxon rank sum test to compare gene expression of cells within the cluster of interest to all cells outside that cluster as implemented by the FindMarkers function. Specific parameters for the genes to be analyzed based on their log fold change between the two compared groups and percent of cells expressing the gene in at least one of the groups are available in the data analysis code. Uniform Manifold Approximation and Projection was used to reduce the PCs to two dimensions for data visualization using the RunUMAP function.

\section{Cell-type identification}

Following PCA and UMAP, we analyzed cluster-specific differential gene expression. Marker genes were plotted using an expression cutoff to facilitate the 
visualization of both high- and low-expression genes on a single plot. Cutoffs are applied so that only cells with expression >cutoff received the color corresponding to that gene. These cutoffs are available in the data analysis code.

\section{Harmony analysis}

To merge the previously published WT dataset with the tumor dataset, we used the Harmony algorithm [53]. First, the WT and tumor dataset were analyzed in single SCTransform normalization and PCA steps. The Harmony algorithm then used the cells' PCA coordinates and dataset identity to calculate new coordinates for each cell so as to minimize dataset dependence when applying clustering to the cells. This algorithm produced a dimensional reduction that was used in place of PCA with the same steps applied to the data as described in the "Data normalization, clustering, differential gene expression, and visualization" and "Cell-type identification" sections of the Methods.

\section{Data Availability}

The scRNA-seq data were deposited in the Gene Expression Omnibus database under the accession code GSE150579 and GSE GSE190297.

\section{Acknowledgements}

We thank the UNC CGBID Histology Core, supported by P30 DK 034987 and the UNC Tissue Pathology Laboratory Core supported by NCI CA016086. T.D. was supported by NINDS (F31 NS120459). T.R.G. was supported by NINDS (R01NS088219, R01NS102627, R01NS106227) and by the UNC Department of Neurology Research 
Fund, and by a TTSA grant from the NCTRACS Institute, which is supported by the

National Center for Advancing Translational Sciences (NCATS), National Institutes of Health, through Grant Award Number UL1TR002489.

\section{Author Contributions}

T.D., D.S.M., A.T. and T.R.G. wrote the manuscript. T.D., D.S.M. and H.L. conducted the experiments and analyzed the data. 


\section{References}

1. Northcott, P.A., et al., Subgroup-specific structural variation across 1,000 medulloblastoma genomes. Nature, 2012. 488(7409): p. 49-56.

2. Kool, M., et al., Molecular subgroups of medulloblastoma: an international metaanalysis of transcriptome, genetic aberrations, and clinical data of WNT, SHH, Group 3, and Group 4 medulloblastomas. Acta Neuropathologica, 2012. 123(4): p. 473-84.

3. Northcott, P.A., et al., Medulloblastoma comprises four distinct molecular variants. Journal of clinical oncology : official journal of the American Society of Clinical Oncology, 2011. 29(11): p. 1408-14.

4. Cavalli, F.M.G., et al., Intertumoral Heterogeneity within Medulloblastoma Subgroups. Cancer Cell, 2017. 31(6): p. 737-754 e6.

5. Riemondy, K.A., et al., Neoplastic and Immune single cell transcriptomics define subgroup-specific intra-tumoral heterogeneity of childhood medulloblastoma. Neurooncology, 2021. noab135.

6. Hovestadt, V., et al., Resolving medulloblastoma cellular architecture by single-cell genomics. Nature, 2019. 572(7767): p. 74-79.

7. Oliver, T.G., et al., Loss of patched and disruption of granule cell development in a preneoplastic stage of medulloblastoma. Development, 2005. 132(10): p. 2425-2439.

8. Hallahan, A.R., et al., The SmoA1 mouse model reveals that notch signaling is critical for the growth and survival of sonic hedgehog-induced medulloblastomas. Cancer Research, 2004. 64(21): p. 7794-800.

9. Zhang, L., et al., Single-Cell Transcriptomics in Medulloblastoma Reveals Tumor-Initiating Progenitors and Oncogenic Cascades during Tumorigenesis and Relapse. Cancer Cell, 2019. 36(3): p. 302-318 e7.

10. Ocasio, J., et al., scRNA-seq in medulloblastoma shows cellular heterogeneity and lineage expansion support resistance to SHH inhibitor therapy. Nat Commun, 2019. 10(1): p. 5829.

11. Vladoiu, M.C., et al., Childhood cerebellar tumours mirror conserved fetal transcriptional programs. Nature, 2019. 572(7767): p. 67-73.

12. Yao, M., et al., Astrocytic trans-Differentiation Completes a Multicellular Paracrine Feedback Loop Required for Medulloblastoma Tumor Growth. Cell, 2020. 180(3): p. 502520 e19.

13. Hardie, D.G., Minireview: The AMP-Activated Protein Kinase Cascade: The Key Sensor of Cellular Energy Status. Endocrinology, 2003. 144(12): p. 5179-5183.

14. Hardie, D.G., F.A. Ross, and S.A. Hawley, AMP-Activated Protein Kinase: A Target for Drugs both Ancient and Modern. Chemistry \& Biology, 2012. 19(10): p. 1222-1236.

15. Tech, K., M. Deshmukh, and T.R. Gershon, Adaptations of energy metabolism during cerebellar neurogenesis are co-opted in medulloblastoma. Cancer Letters, 2015. 356(2 Pt A): p. 268-72.

16. Gershon, T.R., et al., Hexokinase-2-mediated aerobic glycolysis is integral to cerebellar neurogenesis and pathogenesis of medulloblastoma. Cancer \& Metabolism, 2013. 1. 
17. Tech, K., et al., Pyruvate Kinase Inhibits Proliferation during Postnatal Cerebellar Neurogenesis and Suppresses Medulloblastoma Formation. Cancer Res, 2017. 77(12): p. 3217-3230.

18. Bhatia, B., et al., Mitogenic Sonic hedgehog signaling drives E2F1-dependent lipogenesis in progenitor cells and medulloblastoma. Oncogene, 2011. 30(4): p. 410-22.

19. Eyrich, N.W., et al., Reactive Oxygen Species Signaling Promotes Hypoxia-Inducible Factor 1alpha Stabilization in Sonic Hedgehog-Driven Cerebellar Progenitor Cell Proliferation. Mol Cell Biol, 2019. 39(8).

20. Malhotra, A., et al., Sonic Hedgehog Signaling Drives Mitochondrial Fragmentation by Suppressing Mitofusins in Cerebellar Granule Neuron Precursors and Medulloblastoma. Mol Cancer Res, 2016. 14(1): p. 114-24.

21. Hollstein, P.E., et al., The AMPK-Related Kinases SIK1 and SIK3 Mediate Key TumorSuppressive Effects of LKB1 in NSCLC. Cancer Discov, 2019. 9(11): p. 1606-1627.

22. Galan-Cobo, A., et al., LKB1 and KEAP1/NRF2 Pathways Cooperatively Promote Metabolic Reprogramming with Enhanced Glutamine Dependence in KRAS-Mutant Lung Adenocarcinoma. Cancer Res, 2019. 79(13): p. 3251-3267.

23. Zeng, Q., et al., LKB1 inhibits HPV-associated cancer progression by targeting cellular metabolism. Oncogene, 2017. 36(9): p. 1245-1255.

24. Eichner, L.J., et al., Genetic Analysis Reveals AMPK Is Required to Support Tumor Growth in Murine Kras-Dependent Lung Cancer Models. Cell Metab, 2019. 29(2): p. 285-302 e7.

25. Herzig, S. and R.J. Shaw, AMPK: guardian of metabolism and mitochondrial homeostasis. Nat Rev Mol Cell Biol, 2018. 19(2): p. 121-135.

26. Williams, T., et al., AMP-activated protein kinase (AMPK) activity is not required for neuronal development but regulates axogenesis during metabolic stress. Proceedings of the National Academy of Sciences, 2011. 108(14): p. 5849-5854.

27. Zhang, H., et al., Loss of AMPKalpha2 Impairs Hedgehog-Driven Medulloblastoma Tumorigenesis. Int J Mol Sci, 2018. 19(11).

28. D'Amico, D., et al., Non-canonical Hedgehog/AMPK-Mediated Control of Polyamine Metabolism Supports Neuronal and Medulloblastoma Cell Growth. Dev Cell, 2015. 35(1): p. 21-35.

29. Mao, J., et al., A novel somatic mouse model to survey tumorigenic potential applied to the Hedgehog pathway. Cancer Res, 2006. 66(20): p. 10171-8.

30. Schüller, U., et al., Acquisition of Granule Neuron Precursor Identity Is a Critical Determinant of Progenitor Cell Competence to Form Shh-Induced Medulloblastoma. Cancer Cell, 2008. 14(2): p. 123-134.

31. Malawsky, D.S., et al., Cryptic developmental events determine medulloblastoma radiosensitivity and cellular heterogeneity without altering transcriptomic profile. Communications Biology, 2020. in press.

32. Loo, L.Z., M, Drop seq brain. Nat Commun, 2019.

33. Vladoiu, M.C., et al., Childhood cerebellar tumors mirror conserved fetal transcriptional programs. bioRxiv, 2018: p. 350280.

34. Luecken, M.D. and F.J. Theis, Current best practices in single-cell RNA-seq analysis: $a$ tutorial. Mol Syst Biol, 2019. 15(6): p. e8746. 
35. Villman, K., et al., Topoisomerase II-alpha expression in different cell cycle phases in fresh human breast carcinomas. Mod Pathol, 2002. 15(5): p. 486-91.

36. Schuller, U., et al., Forkhead transcription factor FoxM1 regulates mitotic entry and prevents spindle defects in cerebellar granule neuron precursors. Molecular and Cellular Biology, 2007. 27(23): p. 8259-70.

37. Kalinka, A., The probability of drawing intersections: extending the hypergeometric distribution. arXiv, 2013. 1305.0717.

38. Gwinn, D.M., et al., AMPK phosphorylation of raptor mediates a metabolic checkpoint. Mol Cell, 2008. 30(2): p. 214-26.

39. Corradetti, M.N., et al., Regulation of the TSC pathway by LKB1: evidence of a molecular link between tuberous sclerosis complex and Peutz-Jeghers syndrome. Genes Dev, 2004. 18(13): p. 1533-8.

40. Inoki, K., T. Zhu, and K.L. Guan, TSC2 mediates cellular energy response to control cell growth and survival. Cell, 2003. 115(5): p. 577-90.

41. Stoppani, J., et al., AMP-activated protein kinase activates transcription of the UCP3 and HKII genes in rat skeletal muscle. Am J Physiol Endocrinol Metab, 2002. 283(6): p. E123948.

42. Bhatia, B., et al., Hedgehog-mediated regulation of PPARgamma controls metabolic patterns in neural precursors and shh-driven medulloblastoma. Acta Neuropathologica, 2012. 123(4): p. 587-600.

43. Li, Y.H., et al., AMP-Activated Protein Kinase Directly Phosphorylates and Destabilizes Hedgehog Pathway Transcription Factor GLI1 in Medulloblastoma. Cell Rep, 2015. 12(4): p. 599-609.

44. Zhang, R., et al., Dual degradation signals destruct GLI1: AMPK inhibits GLI1 through beta-TrCP-mediated proteasome degradation. Oncotarget, 2017. 8(30): p. 49869-49881.

45. Kimura, N., et al., A possible linkage between AMP-activated protein kinase (AMPK) and mammalian target of rapamycin (mTOR) signalling pathway. Genes Cells, 2003. 8(1): p. 65-79.

46. Bolster, D.R., et al., AMP-activated protein kinase suppresses protein synthesis in rat skeletal muscle through down-regulated mammalian target of rapamycin (mTOR) signaling. J Biol Chem, 2002. 277(27): p. 23977-80.

47. Wu, C.C., et al., mTORC1-Mediated Inhibition of 4EBP1 Is Essential for Hedgehog Signaling-Driven Translation and Medulloblastoma. Dev Cell, 2017. 43(6): p. 673-688 e5.

48. Crowther, A.J., et al., Radiation Sensitivity in a Preclinical Mouse Model of Medulloblastoma Relies on the Function of the Intrinsic Apoptotic Pathway. Cancer Res, 2016. 76(11): p. 3211-23.

49. Ocasio, J.K., et al., GSK-3 modulates SHH-driven proliferation in postnatal cerebellar neurogenesis and medulloblastoma. Development, 2019. 146(20).

50. Lang, P.Y., et al., ATR maintains chromosomal integrity during postnatal cerebellar neurogenesis and is required for medulloblastoma formation. Development, 2016. 143(21): p. 4038-4052.

51. Macosko, E.Z., et al., Highly Parallel Genome-wide Expression Profiling of Individual Cells Using Nanoliter Droplets. Cell, 2015. 161(5): p. 1202-1214. 
bioRxiv preprint doi: https://doi.org/10.1101/2021.12 09.471978; this version posted December 9,2021 . The copyright holder for this preprint (which was not certified by peer review) is the author/funder, who has granted bioRxiv a license to display the preprint in perpetuity. It is made available under aCC-BY-NC-ND 4.0 International license.

52. Butler, A., et al., Integrating single-cell transcriptomic data across different conditions, technologies, and species. Nat Biotechnol, 2018. 36(5): p. 411-420.

53. Korsunsky, I., et al., Fast, sensitive and accurate integration of single-cell data with Harmony. Nat Methods, 2019. 16(12): p. 1289-1296. 